\title{
Radiation Dose and Image Quality Assessment of the Bolus Timing Method for CT Angiography
}

\author{
Myeong Seong Kim, ${ }^{1}$ Jong-Woong Lee, ${ }^{2}$ Sun Geun Kim, ${ }^{3}$ and Dae Cheol Kweon ${ }^{4,}$ \\ ${ }^{1}$ Department of Radiology, The Korean National Cancer Center, Goyang, Republic of Korea \\ ${ }^{2}$ Department of Radiology, Kyung Hee University Hospital at Gang-dong, Seoul, Republic of Korea \\ ${ }^{3}$ Department of Radiological Technology, Baekseok Culture University, Cheonan, Republic of Korea \\ ${ }^{4}$ Department of Radiological Science, Shinhan University, Uijeongbu, Republic of Korea \\ "Corresponding author: Dae Cheol Kweon, Department of Radiological Science, Shinhan University, Uijeongbu, Republic of Korea. Tel: +82-318703416, Fax: +82-318703419, \\ E-mail: dckweon@gmail.com
}

Received 2015 September 15; Revised 2015 October 21; Accepted 2015 October 29.

\begin{abstract}
Background: Bolus timing monitoring scans used for computed tomography angiography (CTA) repetitively expose the same region of the body to radiation with a view to determining the appropriate threshold attenuation level of the contrast material (CM). Therefore, for an effective CT scan, it is necessary to minimize repetitive radiation exposures from bolus timing.

Objectives: This study aims to effectively identify and reduce the radiation dose needed for repetitive monitoring scans utilized in CT involving the bolus timing method.

Patients and Methods: To investigate the currently employed protocols and the number of monitoring scans in bolus timing CT studies, 983 patients (581 males and 402 females; mean age 62.7) were retrospectively analyzed from October 1, 2013 to March 31, 2014. To identify the appropriate $\mathrm{kVp}$ and $\mathrm{mA}$ change in the hounsfield unit (HU) and measure the contrast-to-noise ratio (CNR) and the signal-to-noise ratio (SNR) in the bolus timing method, a $2 \mathrm{~mL}$ syringe containing CM diluted with saline at a ratio of 1 to 300 was inserted into cavities of a CT dose index (CTDI) head phantom and scanned with a 64-section multi-detector row CT system. The dose variation was analyzed using results from the ionization chamber and CTDI.

Results: This retrospective analysis of bolus-timing CT studies found that patients underwent an average of 4.5 to 5.4 monitoring scans. Furthermore, while the HU value of the mixed CM syringe in the central CTDI head phantom cavity was inversely proportional to $\mathrm{mA}$, the decrease in the HU value became weaker at a value of $50 \mathrm{~mA}$ with $100 \mathrm{kVp}$ and at $30 \mathrm{~mA}$ with $120 \mathrm{kVp}$. Here, the CNR(Pearson correlation $r=0.983$, Kendall's $\mathrm{t}=0.978, \mathrm{P}<0.000$ ) became slightly higher with an increase in $\mathrm{mA}$ at $100 \mathrm{kVp}$ compared to $120 \mathrm{kVp}$, whereas the SNR (Pearson correlation $r=0.997$, Kendall's $\mathrm{t}=1.0, \mathrm{P}<0.000$ ) became similar to $100 \mathrm{kVp}$.

Conclusion: It is recommended that minimum level of radiation (100 kVp, $50 \mathrm{~mA} ; 120 \mathrm{kVp}, 30 \mathrm{~mA})$ at which the HU value at the trigger threshold is not significantly different from that of the actual helical scans should be used to minimize the radiation dose caused by repetitive monitoring scans in the bolus timing method.
\end{abstract}

Keywords: Angiography, Image Analysis, Multidetector Computed Tomography, Radiation Dosage

\section{Background}

The universal use of multi-detector computed tomography (MDCT) in hospitals has recently led to an increased use of CT angiography (CTA) and other dynamic studies (1-7). CTA is noninvasive, generates fewer motion artifacts than magnetic resonance angiography, and can provide highly sensitive images at a low cost (8).

Generally, CTA involves a bolus timing method (test bolus or bolus tracking technique) in which a contrast material (CM) is intravenously injected at a volume that exceeds the trigger threshold $(5,9)$. In addition, a real-time monitoring scan is performed to confirm that the CM has reached the trigger threshold at the intravenous (IV) location of the region of interest (ROI) cursor. Simultaneously, the same region is repetitively exposed to radiation. The number of monitoring scans generally depends on the IV location of the CM, the injection rate, the CM volume, the CM concentration, saline flushing, and patient characteristics (weight and size, central blood volume, blood flow, age, cardiac output, degree of hydration, etc.) $(8,10-13)$. These factors can be used as determinants of the radiation dose when employing the bolus timing method.

Undergoing repetitive, short CT scans has been shown to increase the likelihood of cancer occurrence (14). A cohort study in the United States found that $0.7 \%$ of all patients who had received a CT scan developed cancer (15), and that $1.5 \%$ to $2.0 \%$ may have developed cancer due to a CT scan (16). To reduce concerns about repetitive radiation to the same region during a monitoring scan with the bolus timing method, which is essential for CTA, efforts should be made to minimize the side effects of the radiation dose by properly controlling determinants of the 
number of monitoring scans. It is currently recommended to use $100 \mathrm{kVp}$ rather than $120 \mathrm{kVp}$ to reduce the radiation dose and to raise both the contrast-to-noise ratio (CNR) and the signal-to-noise ratio (SNR) in CTA $(2,17)$.

\section{Objectives}

This study aims to effectively identify and reduce the radiation dose needed for the repetitive monitoring scans utilized in CT involving the bolus timing method.

\section{Patients and Methods}

\subsection{Patients' Bolus Timing}

To analyze the radiation dose exposure resulting from the bolus timing method, we selected 871 scanned cases receiving dynamic CT liver (DCTL) and CTA at the Korean national cancer center between October 1, 2013 and March 31, 2014. Of these, 581 were men, 268 were women, and 22 patients receive more than two scans. The mean age was 62.7 years. All of the subjects had CT scans with the bolus timing method, and the monitoring scans were analyzed for DCTL (450 cases), head and neck CTA (140), pulmonary CTA(140), abdomen CTA (85), and lower extremity CTA (78). The CT device was a 64-section multi-detector row CT system (GE Discovery CT $750 \mathrm{HD}$; GE Healthcare), and the scout scan used $120 \mathrm{kVp}$ and $10 \mathrm{~mA}$. The CT device allowed the same $\mathrm{kVp}$ level to be used in a monitoring scan with bolus timing (Figure 1A). Levels of $120 \mathrm{kVp}$ and $40 \mathrm{~mA}$ were used to analyze the number of monitoring triggers, and the bolus timing protocol presented in Table 1 was applied, as shown in Figure 1A. Neovist 370 contrast agent (Daewoong pharmaceutical Co., Korea) containing iopromide was utilized, and an automatic, dual-shot power injector (auto enhance A50, Nemoto Kyorindo Co., Japan) was used to inject the contrast agent intravenously into patients. This retrospective study did not require institutional review board approval or patient consent.

\subsection{Phantom Bolus Timing}

ACT dose index (CTDI) head phantom (18) has been designated by the center for devices in radiological health of the food and drug administration (FDA) as a criterion for determining the radiation dose in a head CT scan. This was used to measure $\mathrm{kVp}, \mathrm{mA}$, the radiation dose in each exposed region, CNR, and SNR with bolus timing (Figure 1B). The phantom was cylindrical with a diameter of $16 \mathrm{~cm}$; it was made of two types of polymethyl methacrylate (i.e., acrylic, Lucite) and gad multiple holes for placement of an ionization chamber. To measure the radiation dose in the
CTDI head phantom, a calibrated ionization chamber (UNFORS Xi, Raysafe AB, Billda, Sweden) was inserted into each of the outer cavities at the central, $0^{\circ}, 90^{\circ}$, and $180^{\circ}$ positions; no measurements were taken at $270^{\circ}$ because of similar position of the $90^{\circ}$ location (Figure 1C).

A thermo-hygrostat was used to keep the CT scan room at a temperature of $21^{\circ} \mathrm{C}$ and humidity of $45 \%$. The ionization chamber was placed in the room for one hour before measuring the dose to improve accuracy of the measurements. The radiation dose was measured within a range of $10 \mathrm{~mA}$ to $50 \mathrm{~mA}$, increasing in increments of $10 \mathrm{~mA}$ for both $100 \mathrm{kVp}$ and $120 \mathrm{kVp}$. The same protocol for the monitoring scan with bolus timing was applied to the remaining scan conditions.

To examine the change in the CNR and SNR of the CM in response to variation in $\mathrm{kVp}$ and $\mathrm{mA}$, a $2 \mathrm{~mL}$ syringe containing a 1:300 mixture of CM and saline was inserted into the central cavity of the CTDI head phantom (Figure 1D). A monitoring scan was performed in the same manner using a range of $10 \mathrm{~mA}$ to $100 \mathrm{~mA}$, increasing in increments of 10 $\mathrm{mA}$ for both $100 \mathrm{kVp}$ and $120 \mathrm{kVp}$. To reduce Hounsfield unit (HU) and noise errors, the scan was repeated three times to determine the average measurement for each level of $\mathrm{kVp}$. The CTDI was used to examine the dose variation by $\mathrm{kVp}$ and $\mathrm{mA}$.

\subsection{Image Analysis}

To measure the CNR and SNR of the mixed contrast agent in the CTDI head phantom, CT images were sent to AW 4.5 (advantage workstation, GE healthcare, Milwaukee, WI) to measure HU and noise. Measurements were made in the middle of the syringe inserted into the central cavity of the head phantom (CM area) and in the lateral part of the phantom, $1 \mathrm{~cm}$ away from the central cavity (non-CM area). The (background) ROI image size was set at $33 \mathrm{~mm}^{2}$.

CNR and SNR were calculated using the following equations:

$\mathrm{CNR}=(\mathrm{CM} H \mathrm{HU}$ - non-CM HU)/background noise,

$\mathrm{SNR}=\mathrm{CM} \mathrm{HU} /$ background noise.

To determine the mean significant difference of the CNR and SNR at $100 \mathrm{kVp}$ and $120 \mathrm{kVp}$, correlation and twotailed student's t-tests were performed using SPSS for Windows statistical software (SPSS version 20.0 SPSS, IBM, NY, USA).

\section{Results}

Slight variation was found among the different cavities. The smallest dose was found in the central cavity of the CTDI head phantom, with increasing doses found in the $180^{\circ}, 90^{\circ}$, and $0^{\circ}$ cavities for both levels of tube voltage 
Table 1. Use of DCTL and CTA Protocols for the Bolus Timing Method

\begin{tabular}{|c|c|c|}
\hline & Parameter & Value \\
\hline \multirow{2}{*}{ Scout } & Tube voltage (kVp) & 120 \\
\hline & Tube current, mA & 10 \\
\hline \multirow{6}{*}{$\begin{array}{l}\text { Automatic bolus tracking } \\
\text { protocol }\end{array}$} & Monitoring scan, mA & 40 \\
\hline & ROI cursor $\left(10-15 \mathrm{~mm}^{2}\right)$ & $\begin{array}{l}\text { DCTL: abdominal aorta (dome of liver level); head and neck CTA: } \\
\text { aortic arch; Pulmonary CTA: ascending aorta; Abdomen CTA: } \\
\text { descending aorta (mid-liver level); Lower extremity CTA: common } \\
\text { iliac artery }\end{array}$ \\
\hline & Monitoring delay (sec) & $\begin{array}{l}\text { DCTL: 16; Head and neck CTA: 6.7; Pulmonary CTA: 5.3; Abdomen } \\
\text { CTA:10; Lower extremity CTA: } 12.8\end{array}$ \\
\hline & Monitoring inter scan delay (sec) & $\begin{array}{l}\text { DCTL: 3; head and neck CTA: 1; Pulmonary CTA: 3; Abdomen CTA: } 3 \text {; } \\
\text { Lower extremity CTA: } 2.5\end{array}$ \\
\hline & Diagnostic delay (sec) & $\begin{array}{l}\text { DCTL: } 16 \text {; Head and neck CTA: } 6.7 \text {; Pulmonary CTA: 10; Abdomen } \\
\text { CTA: } 10 \text {; Lower extremity CTA: } 12.8\end{array}$ \\
\hline & Enhancement threshold (HU) & 100 \\
\hline
\end{tabular}

Abbreviations: DCTL, dynamic CT liver; CTA, computed tomography angiography; ROI, region of interest.
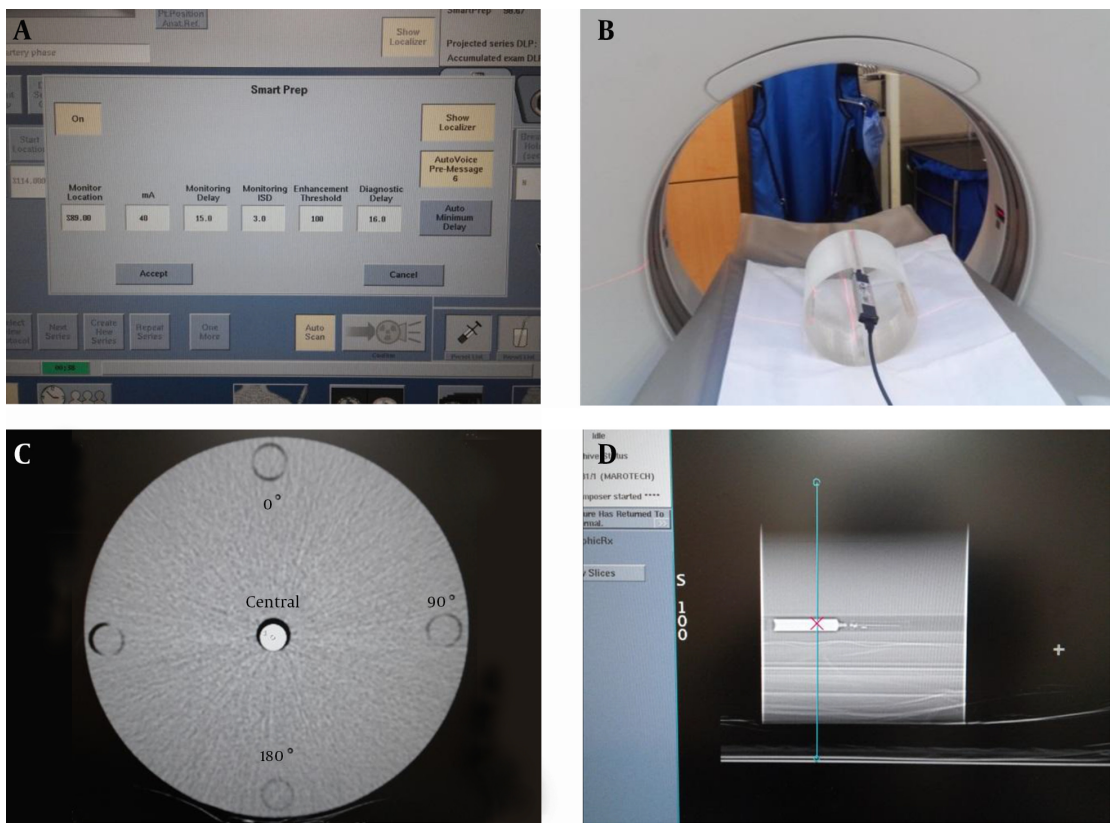

Figure 1. Images of the bolus timing protocol with the CTDI head phantom. A, Imaging was carried out with the bolus timing protocol on the CT scanner; B, An ionization chamber was inserted into the CTDI head phantom cavity for the radiation dose; C, CTDI head phantom CT image; CM and saline were mixed in a 2 mL syringe and inserted into the central cavity of the CTDI head phantom; D, Setting of the ROI position of the mixed CM syringe for bolus monitoring (Abbreviations: CTDI, computed tomography dose index; CM, contrast material; ROI, region of interest).

(100 kVp and $120 \mathrm{kVp}$; Figure 2). Table 2 shows the detailed results of radiation dose at different positions for both 100 $\mathrm{kVp}$ and $120 \mathrm{kVp}$. When the voltage was shifted from 100 $\mathrm{kVp}$ to $120 \mathrm{kVp}$, the dose decreased at a higher rate in the central cavity (63.18\% reduction; $r=0.995$; paired t-test $\mathrm{P}$ Value $=0.03)$ than in the $0^{\circ}$ cavity $(53.16 \%$ reduction; $r=$
0.996; paired t-test $\mathrm{P}$ Value $=0.13$ )

CTA exam analysis showed that the mean number of monitoring scans per patient ranged from 4.5 to 5.4, with deviation ranging from \pm 1.4 to 2.5 (Table 3 ). The smallest number of monitoring scans was performed in DCTL, and the largest number of radiation exposures was found 
A

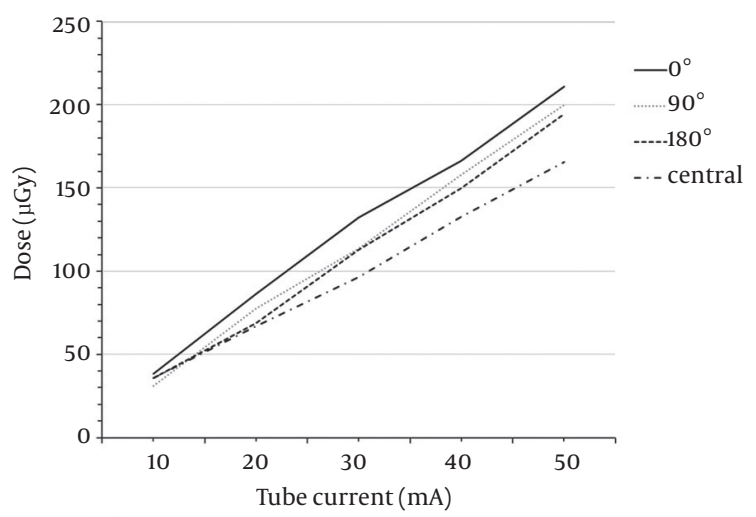

B

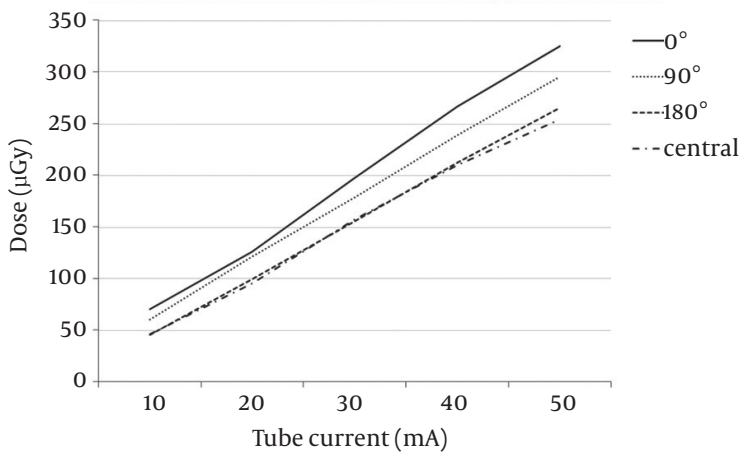

Figure 2. . Comparison of radiation dose depending on variation in angular positioning of the CTDI head phantom using ionization chamber dosimetry. A, The radiation dose depending on variation in angular positioning of CTDI head phantom using ionization chamber dosimetry at $100 \mathrm{kVp}$; B, The radiation dose depending on variation in angular position of the CTDI head phantom using ionization chamber dosimetry at $120 \mathrm{kVp}$ (Abbreviation: CTDI, computed tomography dose index).

in lower extremity angiographies (Table 3 ).

To examine $\mathrm{HU}$ variation in response to changes in $\mathrm{kVp}$ and $\mathrm{mA}$ during monitoring scans, a mixed $\mathrm{CM}$ syringe was inserted into the central cavity of the CTDI head phantom. It was found that the HU value was inversely proportional to the $\mathrm{mA}$ value, with a gap of approximately $25 \mathrm{HU}$, when a voltage of $100 \mathrm{kVp}$ rather than $120 \mathrm{kVp}$ was applied (Figure 3). When $100 \mathrm{kVp}$ was used, the HU value decreased drastically until it reached $40 \mathrm{~mA}$; it then began to decrease gradually at $50 \mathrm{~mA}$. When $120 \mathrm{kVp}$ was used, the HU value decreased drastically until it reached $20 \mathrm{~mA}$ and then began to lessen gradually. The CNR and SNR were used as image evaluation indices, and as shown in Figure 4, the increase in $\mathrm{kVp}$ and $\mathrm{mA}$ led to a decrease in $\mathrm{HU}$ and background noise. When $120 \mathrm{kVp}$ was used, $\mathrm{HU}$ and noise decreased markedly between $10 \mathrm{~mA}$ and $20 \mathrm{~mA}$ and then began to decrease gradually at $30 \mathrm{~mA}$. When $100 \mathrm{kVp}$ was used, $\mathrm{HU}$ and noise decreased drastically until $30 \mathrm{~mA}$ and then be- gan to decrease gradually at $40 \mathrm{~mA}$. Consequently, at 100 $\mathrm{kVp}$ rather than $120 \mathrm{kVp}$, the increase of $\mathrm{mA}$ led to significant variation in the CNR (Figure 4A) but only slight variation in the SNR (Figure 4B).

The correlation test showed that CNR varied in a statistically significant manner (Pearson correlation $\mathrm{r}=0.983$, Kendall's $\mathrm{t}=0.978, \mathrm{P}<0.000$ ), while SNR showed similar results between $100 \mathrm{kVp}$ and $120 \mathrm{kVp}$ (Pearson correlation $r=0.997$, Kendall's $t=1.0, P<0.000)$.

\section{Discussion}

Bolus timing methods can be divided into either test bolus or bolus tracking techniques. A test bolus technique may be performed with fixed delay by injecting a small volume (approx. $5 \mathrm{~mL}$ ) of CM before a CT scan to identify the peak HU. This makes it possible to determine the exact time of maximum peak enhancement for the trigger threshold HU prior to performing a CT scan; however, the HU value based solely on the venous system without considering the saline chaser and $\mathrm{CM}$ volume of the bolus can create variation in the actual CT scan (5, 9). In contrast, a bolus tracking technique makes it possible to track the CM coming into vessels while the $\mathrm{CT}$ scan is being performed and a timing scan of the trigger threshold $\mathrm{HU}$ can be performed using a relatively smaller volume of $\mathrm{CM}(5,9,10)$. Generally, the radiation emission of the CT X-ray tube is affected by the $\mathrm{mA}$, time, and $\mathrm{kVp}$.

The bolus timing method necessary for a CTA scan can cause repetitive radiation exposure due to the monitoring scan that is used to identify the $\mathrm{HU}$ value of $\mathrm{CM}$. As seen in Table 3, the radiation dose by monitoring scan was higher in the lower extremity CTA than in the DCTL study. This is likely because the average number of monitoring scans in lower extremity CTA was approximately one greater than

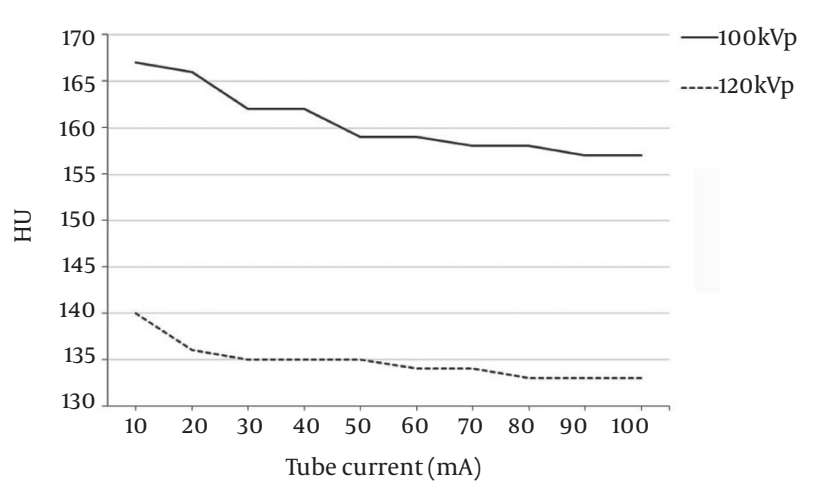

Figure 3. The relationship between $\mathrm{mA}$ and $\mathrm{HU}$ in the mixed $\mathrm{CM}$ syringe inserted into the central cavity of the CTDI head phantom (Abbreviations: CTDI, computed tomography dose index; $\mathrm{CM}$, contrast media; HU, Hounsfield unit) 
Table 2. Radiation Dose ( $\mu \mathrm{Gy}$ ) Based on Angular Positioning of CTDI Head Phantom Using Ionization Chamber Dosimetry

\begin{tabular}{|c|c|c|c|c|c|}
\hline \multirow{2}{*}{$\begin{array}{l}\text { Tube Voltage, } \\
\text { kV }\end{array}$} & \multirow[t]{2}{*}{ Tube Current, mA } & \multicolumn{4}{|c|}{ Cavity Position } \\
\hline & & Central, $\mu \mathbf{G y}$ & $\mathbf{1 8 0}^{\circ}, \mu \mathbf{G y}$ & $\mathbf{9 0}^{\circ}, \mu \mathbf{G y}$ & $\mathbf{0}^{\circ}, \mu \mathbf{G y}$ \\
\hline \multirow{5}{*}{$100 / 120$} & 10 & $35.7 / 46$ & $35.7 / 45.3$ & $31.0 / 60.3$ & $38.4 / 69.9$ \\
\hline & 20 & $66.9 / 86$ & $68.8 / 110.2$ & $77.3 / 121.1$ & $86.4 / 125.6$ \\
\hline & 30 & $96.3 / 156.9$ & $113.1 / 155.3$ & $113.8 / 178$ & $132.2 / 197$ \\
\hline & 40 & $132.8 / 209.6$ & $130.8 / 212.1$ & $166.4 / 238.5$ & $166.2 / 266.4$ \\
\hline & 50 & $165.4 / 254$ & $194.3 / 264.8$ & $182.1 / 295.4$ & $210.9 / 324.8$ \\
\hline $\begin{array}{l}\text { P Value } \\
\text { (2-tailed) }\end{array}$ & & 0.03 & 0.018 & 0.011 & 0.013 \\
\hline
\end{tabular}

Abbreviations: CTDI, computed tomography dose index.

Table 3. The Mean Number of Monitoring Scans Performed in Patients With the Bolus Timing Method

\begin{tabular}{l|l|c|c}
\hline \multicolumn{2}{c|}{ Exams } & $\begin{array}{c}\text { Intravenous Flow Rate (Contrast Agent: } \\
\text { Saline; mL/sec) }\end{array}$ & $\begin{array}{c}\text { No. of Average Monitor (Exposure of } \\
\text { Radiation) }\end{array}$ \\
\hline \multirow{2}{*}{ Dynamic study } & Liver & $3.4: 3.4$ & 4.5 \\
\hline \multirow{4}{*}{ Angiography } & Head and neck & $4.5: 4.5$ & 4.2 \\
\cline { 2 - 4 } & Pulmonary & $4.0: 4.0$ & 4.6 \\
\cline { 2 - 4 } & Abdomen & $4.0: 4.0$ & 4.7 \\
\cline { 2 - 4 } & Lower extremity & $4.0: 4.0$ & 5.4 \\
\hline
\end{tabular}

in the DCTL study. Since bolus timing method protocols may vary based on hospital standards, monitoring scan outcomes may also be affected. It is therefore necessary to reduce the radiation dose by setting a proper protocol based on the CT study, the patient's characteristics, and properties of the CM.

A CT scan using the bolus tracking technique begins after a time delay following repetitive exposure to radiation until the trigger threshold HU with a fixed level of $\mathrm{kVp}$ and $\mathrm{mA}$ is reached. Thus, when CTA using the bolus tracking technique is performed on a patient with vascular stenosis or an occlusion in the scan region, it is necessary to avoid unnecessary radiation exposure by decreasing the number of monitoring scans and increasing the monitoring delay. This is necessary because of the decreased blood flow in these patients (10). Such patient characteristics as weight, size, central blood volume, blood flow, age, and cardiac output should be taken into account, in addition to vascular stenosis. It is also necessary to properly determine the monitoring delay and scan delay based on the saline flush chaser and the CM injection rate, volume, and concentration $(7-13,19,20)$. If a scan is performed without taking these factors into account, especially when using a CTA protocol in a patient whose lower extremity is connected intravenously via the median cubital vein, inconsistencies in the time for the CM to reach the target vein can result, leading to increased radiation exposure. Figure 5 demonstrates the contrast bolus transit time, optimal monitoring phase, and scan time after bolus timing injection in full detail.

The ideal protocol setting for a monitoring scan with bolus timing depends on the HU value in the target vein (awareness of the HU value at the trigger threshold). Use of a $120 \mathrm{kVp}$ is standard in CT imaging studies for patients with a normal body type, as it produces better quality images than any other tube voltage value (2). In CTA or dynamic study CT scans with quick CM injection, the standard tube voltage can lead to images with high CNR and SNR in the ROI and can reduce the radiation dose $(1,2,21)$. As shown in Figure 3, the HU value decreased with an increase in mA(100 kVp: $40 \mathrm{~mA} ; 120 \mathrm{kVp}: 20 \mathrm{~mA})$, but it began to change insignificantly at a certain $\mathrm{mA}$ value $(100 \mathrm{kVp}$ : $50 \mathrm{~mA} ; 120 \mathrm{kVp}: 30 \mathrm{~mA}$ ). At a constant $\mathrm{mA}$ value, the $\mathrm{HU}$ value depended more heavily on the $\mathrm{kVp}$ level (9). Background noise exhibited a similar correlation with $\mathrm{mA}$ to that of the HU value. That is, when $100 \mathrm{kVp}$ was used, background noise decreased drastically until a value of $40 \mathrm{~mA}$ was reached (120 kVp: $20 \mathrm{~mA})$, while it began to decrease gradually at $50 \mathrm{~mA}(120 \mathrm{kVp}: 30 \mathrm{~mA})$. To obtain accurate CTA images, it is important to determine an effective scan delay 
A

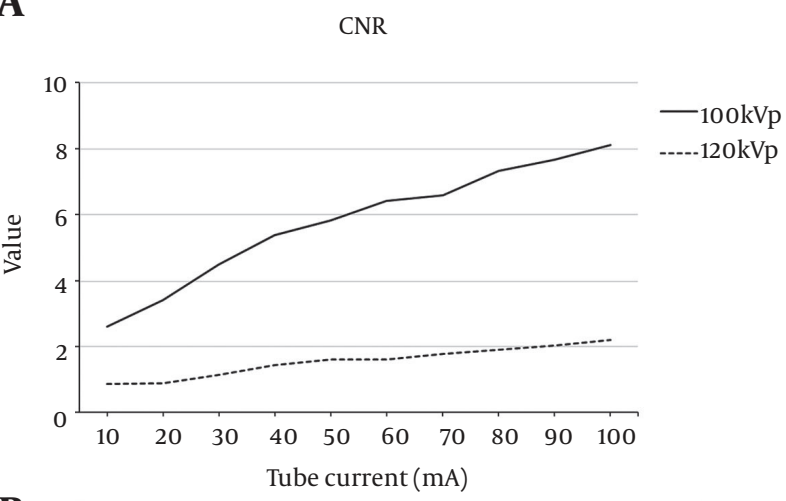

B

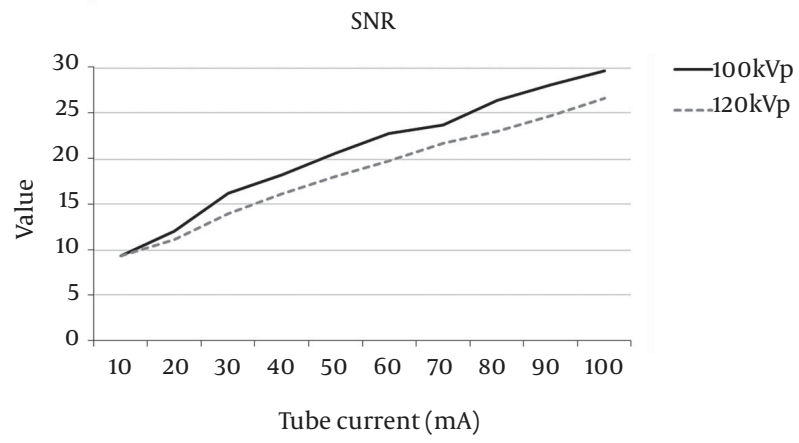

Figure 4. Comparison of the quantitative image quality of the CTDI head phantom central cavity. A, Comparison of the CNRs between the mixed CM syringe and nonCM area of the CTDI head phantom central cavity; B, Comparison of the SNRs in the mixed CM syringe of the CTDI head phantom central cavity (Abbreviations: CTDI, computed tomography dose index; CM, contrast media; CNR, contrast-to-noise ratio; SNR, signal-to-noise ratio).

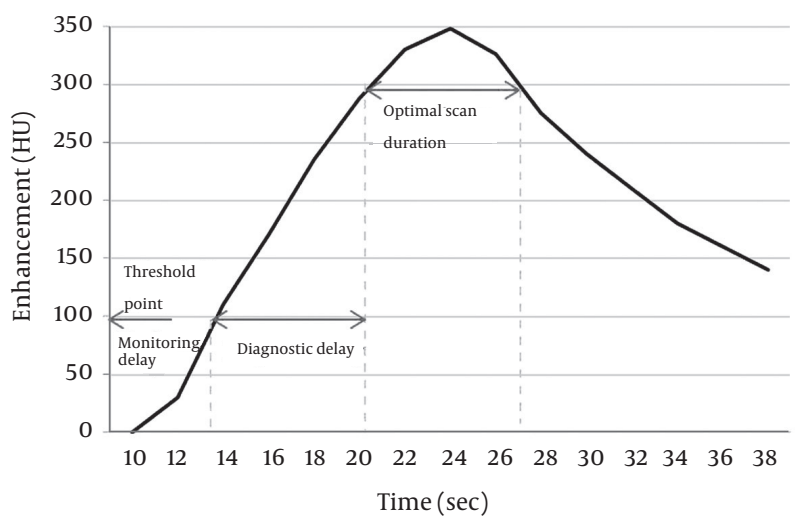

Figure 5. The flow of contrast bolus transit time using bolus-timing injection. The bolus timing method allows the optimal monitoring phase and scan time to be determined and the repetitive radiation dose to be decreased.

duration when the $\mathrm{CM}$ reaches the peak of maximum enhancement during the actual CTA. Therefore, the level of
$\mathrm{mA}$ in a monitoring scan should be determined from the point of inflection of the HU value based on the level of $\mathrm{kVp}$ (100 kVp: $50 \mathrm{~mA}$; $120 \mathrm{kVp:} 30 \mathrm{~mA}$ ). Since this outcome is based on the use of a CTDI head phantom, a patient who weighs more may require a higher level of $\mathrm{mA}$.

The CTDI is a unit related to the energy of the diagnostic X-ray CT scanners for the patient. Generally, the CTDI depends on the $\mathrm{kVp}$ and $\mathrm{mA}$ chosen to perform the CT study (22). In this study, with an increasing $\mathrm{mA}$, the radiation dose was found to be largest in the central cavity of the CTDI head phantom using an ionization chamber, with decreasing values in the $180^{\circ}, 90^{\circ}$, and $0^{\circ}$ cavities (Figure 2). However, at a voltage of $120 \mathrm{kVp}$, there was greater variation in the radiation dose in the central cavity of the CTDI head phantom. Thus, when the tube voltage is lowered from $120 \mathrm{kVp}$ to $100 \mathrm{kVp}$, the radiation dose can be decreased by $53.16 \%$ in the $0^{\circ}$ cavity and by $63.18 \%$ in the central cavity. At $100 \mathrm{kVp}$, the radiation dose was estimated to be $649 \mu \mathrm{Gy}$ in the central cavity and $310 \mu \mathrm{Gy}$ in the $0^{\circ}$ cavity. At $120 \mathrm{kVp}$, it was estimated to be $1,024 \mu \mathrm{Gy}$ in the central cavity and 1,298 $\mu \mathrm{Gy}$ in the $0^{\circ}$ cavity, based on results from the monitoring scan in the head phantom at this hospital (4.88 monitoring scans on average; $40 \mathrm{~mA}$ for the entire scan). This shows that the increase in $\mathrm{kVp}$ and the number of monitoring scans led to a drastically greater amount of radiation exposure.

The international commission on radiological protection (ICRP) reports that organs with relatively higher tissue-weighting factors for the effective dose are located in the $0^{\circ}$ part of the human body and include the lungs, coronary arteries, thyroid, and breasts $(23,24)$. The highest dose was also found at the $0^{\circ}$ position of the head phantom in the experiment, as shown in Figure 2. It is therefore necessary to minimize the number of repetitive monitoring scans in organs such as the lungs, coronary arteries, thyroid, and breasts, as they have higher tissue-weighting factors and are located in the $0^{\circ}$ part of the human body.

In the bolus tracking technique, helical CT scans have typically been performed by pressing the start button after the operator has confirmed that the CM has reached the trigger threshold via a real-time monitoring scan. At present, however, a scan is automatically performed when CM reaches the trigger threshold without the operator's confirmation. Thus, when a bone with high X-ray attenuation or a metal substance is present around an ROI cursor for bolus tracking, dark or streak artifacts can be generated due to beam hardening effects (25). Although in some cases, the motion artifacts caused by breathing can prevent the $\mathrm{CM}$ from coming into the vein, the artifacts may also cause the $\mathrm{HU}$ value to exceed the trigger threshold, leading to a helical CT scan (26). When artifacts are likely to be generated around the target vein for an ROI cursor, it 
is necessary to locate the ROI in another vein and to make the ROI as small as possible so that it is not pushed out of the vein when the patient breathes.

This study has the limitation that it failed to prospectively show variation in the real-time monitoring radiation dose ( $\mathrm{kVp}$ and $\mathrm{mA}$ ) and did not describe how those variations affect the final images. For example, it did not identify the effects of factors like the intravenous CM injection spot, injection rate, CM volume, CM concentration, saline flushing, or patient characteristics, such as weight and size, central blood volume, blood flow, age, cardiac output, and degree of hydration, on monitoring scans with different values of $\mathrm{kVp}$ and $\mathrm{mA}$, and consequently the CTA image. Furthermore, the results were collected from monitoring scans using a bolus tracking protocol specific to the Korean national cancer center. Thus, variations in the trigger threshold value (100), monitoring delay, scan delay, $\mathrm{kVp}, \mathrm{mA}$, patient distribution, IV line gauge, CTA scan type, and CM type could lead to a number of different monitoring scan results. Another limitation is that only a head phantom was used instead of utilizing multiple types of phantoms. This limited the study's ability to show an association between variation in the radiation dose and bolus tracking for each body part.

When the bolus timing method was used in CTA exams, patients are generally exposed to excessive radiation due to repetitive monitoring scans. Thus, it is necessary to use the minimum voltage level $100 \mathrm{kVp}, 50 \mathrm{~mA} ; 120 \mathrm{kVp}, 30 \mathrm{~mA}$ at which the HU value at the trigger threshold is not significantly different from that in actual helical scans. When using a voltage of $100 \mathrm{kVp}$ on deep parts of the body, it was found that the radiation dose decreased at a higher rate, while the CNR and SNR were both slightly higher.

\section{Footnotes}

Authors' Contributions: The study concepts were designed by Dae Cheol Kweon, Myeong Seong, Kim and and Jong Woong Lee; definition of intellectual contents and, literature were performed by Myeong Seong, Kim and and Jong Woong Lee; statistical data analysis and, drafting of the manuscript were col-lected by Myeong Seong, Kim and and Jong Woong Lee; critical revision of the manuscript for important intellectual content were performed by Dae Cheol Kweon and Myeong Seong Kim; study supervision was performed by Dae Cheol Kweon.

Financial Disclosure: None declared.

Funding/Support: Authors who have no relevant financial interests are asked to provide a statement indicating that they have no financial interests related to the material in the manuscript.

\section{References}

1. Wintersperger B, Jakobs T, Herzog P, Schaller S, Nikolaou K, Suess C, et al. Aorto-iliac multidetector-row CT angiography with low $\mathrm{kV}$ settings: improved vessel enhancement and simultaneous reduction of radiation dose. Eur Radiol. 2005;15(2):334-41. doi:10.1007/s00330-0042575-y. [PubMed: 15611872].

2. Heyer CM, Mohr PS, Lemburg SP, Peters SA, Nicolas V. Image quality and radiation exposure at pulmonary CT angiography with 100- or $120-\mathrm{kVp}$ protocol: prospective randomized study. Radiology. 2007;245(2):577-83. doi: 10.1148/radiol.2452061919. [PubMed: 17940308].

3. Napoli A, Fleischmann D, Chan FP, Catalano C, Hellinger JC, Passariello R, et al. Computed tomography angiography: state-of-the-art imaging using multidetector-row technology. J Comput Assist Tomogr. 2004;28 Suppl 1:S32-45. [PubMed: 15258492].

4. Smith-Bindman R, Lipson J, Marcus R, Kim KP, Mahesh M, Gould R, et al. Radiation dose associated with common computed tomography examinations and the associated lifetime attributable risk of cancer. Arch Intern Med. 2009;169(22):2078-86. doi: 10.1001/archinternmed.2009.427. [PubMed: 20008690].

5. Cademartiri F, Nieman K, van der Lugt A, Raaijmakers RH, Mollet $\mathrm{N}$, Pattynama PM, et al. Intravenous contrast material administration at 16-detector row helical CT coronary angiography: test bolus versus bolus-tracking technique. Radiology. 2004;233(3):817-23. doi: 10.1148/radiol.2333030668. [PubMed: 15516601].

6. Fleischmann D. Use of high concentration contrast media: principles and rationale-vascular district. Eur J Radiol. 2003;45 Suppl 1:S88-93. [PubMed: 12598032].

7. Foley WD, Karcaaltincaba M. Computed tomography angiography: principles and clinical applications. J Comput Assist Tomogr. 2003;27 Suppl 1:S23-30. [PubMed: 12908709].

8. Nakajima Y, Yoshimine T, Yoshida H, Sakashita K, Okamoto M, Kishikawa $\mathrm{M}$, et al. Computerized tomography angiography of ruptured cerebral aneurysms: factors affecting time to maximum contrast concentration. J Neurosurg. 1998;88(4):663-9. doi: 10.3171/jns.1998.88.4.0663. [PubMed: 9525712].

9. McCollough CH, Leng S, Yu L, Cody DD, Boone JM, McNitt-Gray MF. CT dose index and patient dose: they are not the same thing. Radiology. 2011;259(2):311-6. doi: 10.1148/radiol.11101800. [PubMed: 21502387].

10. Cademartiri F, van der Lugt A, Luccichenti G, Pavone P, Krestin GP. Parameters affecting bolus geometry in CTA: a review. J Comput Assist Tomogr. 2002;26(4):598-607. [PubMed:12218827].

11. Sandstede JJ, Tschammler A, Beer M, Vogelsang C, Wittenberg G, Hahn D. Optimization of automatic bolus tracking for timing of the arterial phase of helical liver CT. Eur Radiol. 2001;11(8):1396-400. doi: 10.1007/s003300000816. [PubMed: 11519548].

12. Awai K, Hori S. Effect of contrast injection protocol with dose tailored to patient weight and fixed injection duration on aortic and hepatic enhancement at multidetector-row helical CT. Eur Radiol. 2003;13(9):2155-60. doi: 10.1007/s00330-003-1904-x. [PubMed: 12736754].

13. Bae KT, Heiken JP, Brink JA. Aortic and hepatic peak enhancement at CT: effect of contrast medium injection rate-pharmacokinetic analysis and experimental porcine model. Radiology. 1998;206(2):455-64. doi: 10.1148/radiology.206.2.9457200. [PubMed: 9457200].

14. Kim T, Murakami T, Takahashi S, Tsuda K, Tomoda K, Narumi $Y$, et al. Effects of injection rates of contrast material on arterial phase hepatic CT. AJR Am J Roentgenol. 1998;171(2):429-32. doi: 10.2214/ajr.171.2.9694469. [PubMed: 9694469].

15. Zondervan RL, Hahn PF, Sadow CA, Liu B, Lee SI. Frequent body CT scanning of young adults: indications, outcomes, and risk for radiation-induced cancer. J Am Coll Radiol. 2011;8(7):501-7. doi: 10.1016/j.jacr.2010.12.025. [PubMed: 21723488]. 
16. Sodickson A, Baeyens PF, Andriole KP, Prevedello LM, Nawfel RD, Hanson R, et al. Recurrent CT, cumulative radiation exposure, and associated radiation-induced cancer risks from CT of adults. Radiology. 2009;251(1):175-84. doi:10.1148/radiol.2511081296. [PubMed:19332852].

17. Brenner DJ, Hall EJ. Computed tomography-an increasing source of radiation exposure. $N$ Engl J Med. 2007;357(22):2277-84. doi: 10.1056/NEJMra072149. [PubMed: 18046031].

18. Schueller-Weidekamm C, Schaefer-Prokop CM, Weber M, Herold CJ, Prokop M. CT angiography of pulmonary arteries to detect pulmonary embolism: improvement of vascular enhancement with low kilovoltage settings. Radiology. 2006;241(3):899-907. doi: 10.1148/radiol.2413040128. [PubMed: 17114631].

19. Behrendt FF, Bruners P, Keil S, Plumhans C, Mahnken AH, Das M, et al. Effect of different saline chaser volumes and flow rates on intravascular contrast enhancement in CT using a circulation phantom. Eur J Radiol. 2010;73(3):688-93. doi: 10.1016/j.ejrad.2009.01.008. [PubMed: 19349133].

20. Szucs-Farkas Z, Strautz T, Patak MA, Kurmann L, Vock P, Schindera ST. Is body weight the most appropriate criterion to select patients eligible for low-dose pulmonary CT angiography? Analysis of objective and subjective image quality at $80 \mathrm{kVp}$ in 100 patients. Eur Radiol. 2009;19(8):1914-22. doi: 10.1007/s00330-009-1385-7. [PubMed: 19333605].

21. Haage P, Schmitz-Rode T, Hubner D, Piroth W, Gunther RW. Reduction of contrast material dose and artifacts by a saline flush using a double power injector in helical CT of the thorax. AJR
Am J Roentgenol. 2000;174(4):1049-53. doi: 10.2214/ajr.174.4.1741049. [PubMed: 10749248].

22. Huda W, Mettler FA. Volume CT dose index and dose-length product displayed during CT: what good are they?. Radiology. 2011;258(1):23642. doi: 10.1148/radiol.10100297. [PubMed: 20971777].

23. Christner JA, Kofler JM, McCollough $\mathrm{CH}$. Estimating effective dose for CT using dose-length product compared with using organ doses: consequences of adopting International Commission on Radiological Protection publication 103 or dual-energy scanning. AJR Am J Roentgenol. 2010;194(4):881-9. doi: 10.2214/AJR.09.3462. [PubMed: 20308486].

24. Boetticher H, Lachmund J, Looe HK, Hoffmann W, Poppe B. 2007 recommendations of the ICRP change basis for estimation of the effective dose: what is the impact on radiation dose assessment of patient and personnel?. Rofo. 2008;180(5):391-5. doi: 10.1055/s-2008-1027284. [PubMed: 18438741].

25. Yanaga Y, Awai K, Nakaura T, Utsunomiya D, Funama Y, Date S, et al. Hepatocellular carcinoma in patients weighing $70 \mathrm{~kg}$ or less: initial trial of compact-bolus dynamic CT with low-dose contrast material at $80 \mathrm{kVp}$. AJR Am J Roentgenol. 2011;196(6):1324-31. doi: 10.2214/AJR.10.4545. [PubMed: 21606296].

26. Hoffmann MH, Shi H, Manzke R, Schmid FT, De Vries L, Grass M, et al. Noninvasive coronary angiography with 16-detector row $\mathrm{CT}$ : effect of heart rate. Radiology. 2005;234(1):86-97. doi: 10.1148/radiol.2341031408. [PubMed: 15550373]. 